07

\title{
Простой численный метод определения энергетического спектра носителей заряда в полупроводниковых гетероструктурах
}

\author{
(ㄷ Г.Ф. Глинский
}

Санкт-Петербургский государственный электротехнический университет „ЛЭТИ“, Санкт-Петербург, Россия

E-mail: genaglinskii@mail.ru

\section{Поступило в Редакцию 8 ноября 2017 г.}

Предлагается простой численный метод определения энергетического спектра и волновых функций носителей заряда в полупроводниковых гетероструктуpax (квантовые ямы, нити, точки, сверхрешетки) в приближении эффективной массы. Рассматривается общий случай многозонного $k p$-гамильтониана, соответствующий точке Г зоны Бриллюэна. В основе метода лежит дискретное преобразование Фурье для структур с периодически изменяющимся потенциалом. Для одиночных гетероструктур такая периодичность вводится искусственно. Показано, что в рамках данного подхода эффективный матричный гамильтониан гетероструктуры может быть записан в двух унитарно-эквивалентных $a$ - и $k$-представлениях. В качестве примера рассматривается однозонная $k p$-модель гетероструктуры с одиночной параболической, треугольной и прямоугольной квантовыми ямами. Исследуется влияние интерфейсных $k p$-поправок на поведение огибающих функций вблизи резких гетерограниц.

DOI: 10.21883 /PJTF.2018.06.45763.17113

Одним из наиболее распространенных методов определения энергетического спектра носителей заряда в полупроводниковых гетероструктурах является метод эффективной массы [1]. Однако до недавнего времени не было единого мнения относительно вида эффективного $k p$-гамильтониана гетероструктуры, методов решения уравнения Шредингера, а также граничных условий, накладываемых на огибающие волновые функции на гетерограницах. В работах [2,3] впервые предложен симметрийный подход к определению эффективных $k p$-гамильтонианов как без учета, так и с учетом спина и спин-орбитального взаимодей- 
ствия, основанный на теории инвариантов $[4,5]$. В этих работах с точностью до квадратичных по $\mathbf{k}$ членов были определены $k p$-гамильтонианы для электронов и дырок в гетероструктурах на основе прямозонных полупроводников $\mathrm{A}^{3} \mathrm{~B}^{5}$. Было показано, что наряду с обычными $k p$-членами объемных материалов эффективные $k p$-гамильтонианы гетероструктур содержат дополнительные слагаемые, обусловленные эффектами рассеяния носителей заряда на короткодействующей части интерфейсного потенциала.

В настоящей работе предлагается простой численный метод решения уравнения Шредингера в приближении эффективной массы для произвольных гетероструктур на основе прямозонных полупроводников. Показано, что обычно используемые для этих целей дифференциальные уравнения являются континуальным приближением точных алгебраических уравнений, решение которых сводится к поиску собственных чисел и собственных столбцов матриц конечных размерностей.

Уравнение Шредингера для гетероструктуры в приближении эффективной массы в рамках многозонной модели, соответствующей точке $\Gamma$ зоны Бриллюэна, имеет вид $[2,3]$

$$
\sum_{n, \mathbf{k}^{\prime}} H_{m n}\left(\mathbf{k}, \mathbf{k}^{\prime}\right) F_{n}\left(\mathbf{k}^{\prime}\right)=E F_{m}(\mathbf{k}),
$$

где $H_{m n}\left(\mathbf{k}, \mathbf{k}^{\prime}\right)$ - матрица эффективного $k p$-гамильтониана, $F_{m}(\mathbf{k})-$ огибающие волновые функции в $k$-представлении, которые можно рассматривать как Фурье-образы некоторых функций координат. Поскольку все значения волнового вектора $\mathbf{k}$ ограничены зоной Бриллюэна кристалла, с помощью обратного преобразования Фурье можно восстановить огибающие волновые функции $F_{m}(\mathbf{a})$ только в дискретных точках обычного пространства а - узлах решетки Браве. Таким образом, решетка Браве выступает в роли естественной трехмерной сетки для дискретизации пространства, необходимой для реализации численных методов расчета в рамках приближения эффективной массы.

Рассмотрим наиболее общий вид трехмерной сверхрешетки, состоящей из периодически расположенных в пространстве квантовых точек. В этом случае $\mathbf{k}$ удобно представить в виде суммы $\mathbf{k}=\mathbf{k}_{i}+\mathbf{K}$, где $\mathbf{k}_{i}$ - волновые векторы, пробегающие дискретный ряд значений в зоне Бриллюэна кристалла и представляющие собой векторы обратной сверхрешетки, $\mathbf{K}-$ волновой вектор сверхрешетки, изменяющийся в 


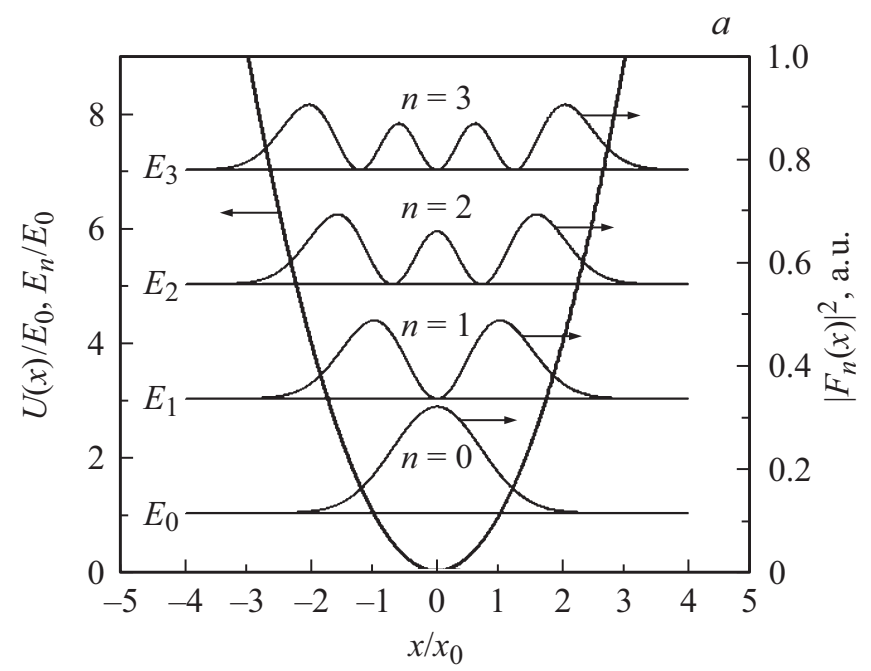

Энергетический спектр и квадрат модуля огибающих волновых функций электрона для четырех нижних состояний $n=0,1,2,3$ в параболической квантовой яме $(a)$, а также для трех нижних состояний $n=0,1,2$ в прямоугольной $(b)$ и треугольной $(c)$ квантовых ямах в GaAs. Данные получены при следующих значениях параметров: $a-D=30 x_{0}, N=100, x_{0}=\sqrt{\hbar / m^{*} \omega}, E_{0}=\hbar \omega / 2$; $b-D=N x_{0}, N=100, x_{0}=a_{0} / 2\left(a_{0}=0.5653 \mathrm{~nm}\right)$, разрыв зоны $\Delta E=0.3 \mathrm{eV}$, ширина ямы $d=40 x_{0}, m^{*}=0.067 m_{0} ; c-$ разрыв зоны на правом интерфейсе $\Delta E=1 \mathrm{eV}$, ширина в основании квантовой ямы $d=40 x_{0}$, остальные параметры совпадают с параметрами, приведенными в подписи к части $b$.

пределах ее зоны Бриллюэна. При этом а удобно записать в виде суммы $\mathbf{a}=\mathbf{D}+\mathbf{a}_{i}$, где $\mathbf{D}$ - произвольный вектор трансляции сверхрешетки, $\mathbf{a}_{i}$ - узлы решетки Браве кристалла, попадающие внутрь сверхъячейки. Наличие трансляционной симметрии у сверхрешетки приводит к тому, что гамильтониан, волновые функции и энергия в правой части уравнения (1) становятся параметрически зависящими от К. В результате уравнение Шредингера принимает вид

$$
\sum_{n, j} H_{m n}\left(\mathbf{k}_{i}, \mathbf{k}_{j} ; \mathbf{K}\right) F_{n}\left(\mathbf{k}_{j} ; \mathbf{K}\right)=E(\mathbf{K}) F_{m}\left(\mathbf{k}_{i} ; \mathbf{K}\right)
$$

2* Письма в ЖТФ, 2018, том 44, вып. 6 

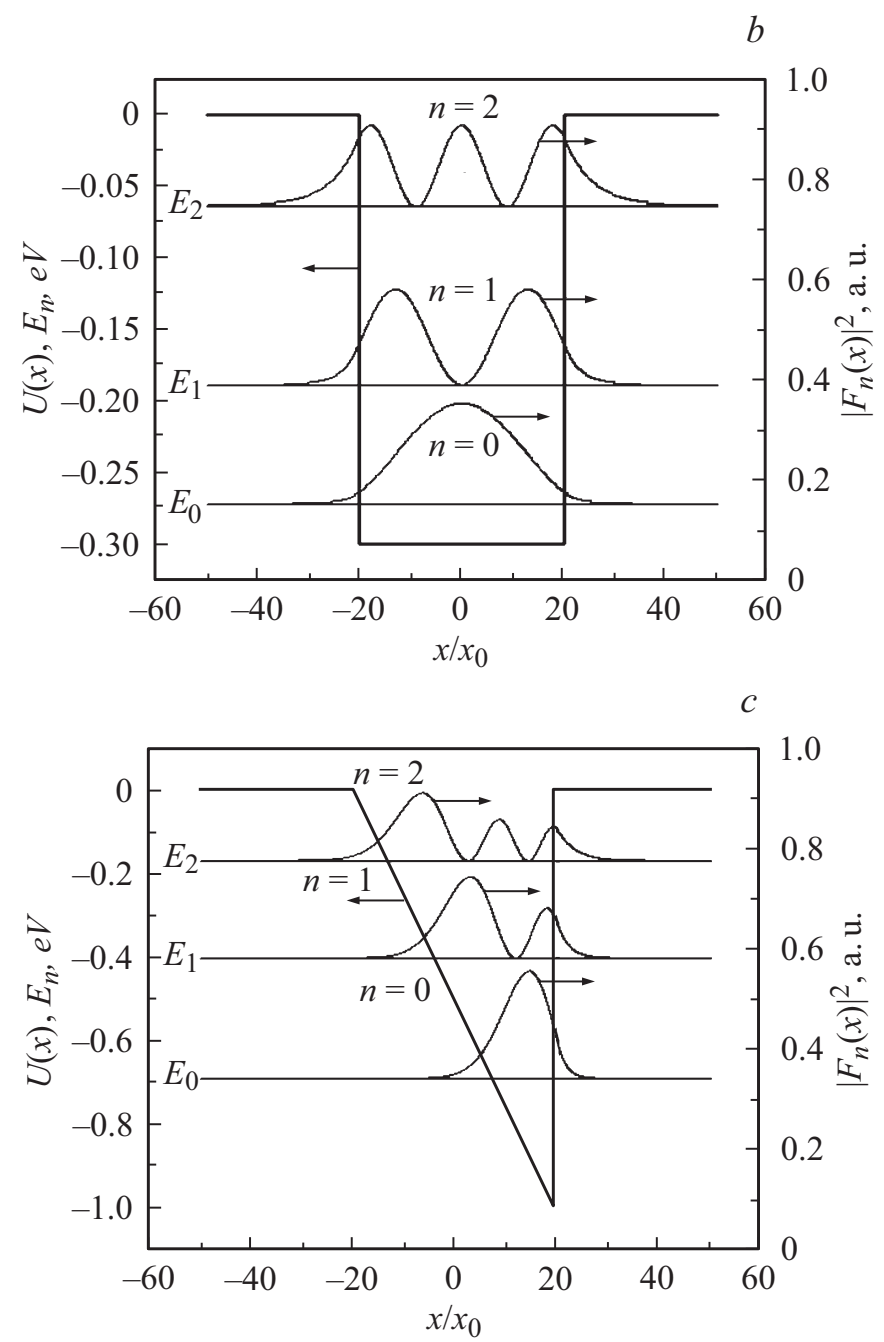

Рисунок (продолжение).

При анализе связанных состояний в одиночных квантовых точках необходимо искусственно вводить сверхрешетку, ширина барьеров

Письма в ЖТФ, 2018, том 44, вып. 6 
которой должна быть увеличена таким образом, чтобы исключить эффекты туннелирования носителей заряда между отдельными сверхъячейками. В этом случае ширина минизон сверхрешетки будет стремиться к нулю, и в уравнении (2) можно положить $\mathbf{K}=0$. В этом приближении уравнение Шредингера для гетероструктуры с одиночной квантовой точкой принимает вид

$$
\sum_{n, j} H_{m n}\left(\mathbf{k}_{i}, \mathbf{k}_{j}\right) F_{n}\left(\mathbf{k}_{j}\right)=E F_{m}\left(\mathbf{k}_{i}\right) .
$$

Это уравнение следует рассматривать как уравнение Шредингера в $k$-представлении. Переход к унитарно-эквивалентному $a$-представлению осуществляется с помощью дискретного преобразования Фурье

$$
\begin{gathered}
F_{m}\left(\mathbf{a}_{i}\right)=\frac{1}{\sqrt{N}} \sum_{j} F_{m}\left(\mathbf{k}_{j}\right) e^{i \mathbf{k}_{\mathbf{j}} \mathbf{a}_{i}}, \\
H_{m n}\left(\mathbf{a}_{i}, \mathbf{a}_{j}\right)=\frac{1}{N} \sum_{i^{\prime}, j^{\prime}} e^{i \mathbf{k}_{i} \mathbf{a}_{i}} H_{m n}\left(\mathbf{k}_{i^{\prime}}, \mathbf{k}_{j^{\prime}}\right) e^{-i \mathbf{k}_{j^{\prime}} \mathbf{a}_{j}},
\end{gathered}
$$

где $N$ - число элементарных ячеек кристалла, содержащихся в сверхъячейке. В результате приходим к следующему уравнению Шредингера в $a$-представлении:

$$
\sum_{n, j} H_{m n}\left(\mathbf{a}_{i}, \mathbf{a}_{j}\right) F_{n}\left(\mathbf{a}_{j}\right)=E F_{m}\left(\mathbf{a}_{i}\right) .
$$

Экспоненты в формулах (3) и (4) играют роль матричных элементов унитарной матрицы $U_{i j}=e^{i \mathbf{k}_{j} \mathbf{a}_{i}}$, удовлетворяющей соотношениям $U^{+} U=U U^{+}=I$ (где $I-$ единичная матрица) и осуществляющей переход от одного представления к другому. Таким образом, решение уравнения Шредингера в обоих представлениях сводится к определению собственных чисел и нормированных на единицу собственных столбцов матричного гамильтониана конечной размерности.

Полная волновая функция носителя заряда в координатном представлении определяется выражением [3]

$$
\psi(\mathbf{x})=\frac{1}{\sqrt{N \Omega}} \sum_{m, i} e^{i \mathbf{k}_{i} \mathbf{x}} F_{m}\left(\mathbf{k}_{i}\right) u_{m}(\mathbf{x}),
$$

где $u_{m}(\mathbf{x})-$ периодическая часть блоховской волновой функции $m$-й зоны в точке $\Gamma, \Omega-$ объем элементарной ячейки кристалла. Если

Письма в ЖТФ, 2018, том 44, вып. 6 
определить огибающую функцию в $x$-представлении как

$$
F_{m}(\mathbf{x})=\frac{1}{\sqrt{N}} \sum_{i} e^{i \mathbf{k}_{i} \mathbf{x}} F_{m}\left(\mathbf{k}_{i}\right),
$$

то приходим к общепринятой форме записи соотношения (5) [1]

$$
\psi(\mathbf{x})=\frac{1}{\sqrt{\Omega}} \sum_{m} F_{m}(\mathbf{x}) u_{m}(\mathbf{x}) .
$$

Однако переход от волновых функций $F_{m}\left(\mathbf{k}_{i}\right)$ к функциям $F_{m}(\mathbf{x})$ в $(6)$ не является унитарным преобразованием, поэтому $F_{m}(\mathbf{x})$ не является решением какого-либо уравнения Шредингера. Только в континуальном пределе, когда $\Omega \rightarrow 0$, а объем зоны Бриллюэна кристалла стремится к бесконечности, эти функции можно приближенно определить, решая уравнение Шредингера в $x$-представлении, которое в рамках многозонной модели является системой дифференциальных уравнений с периодическими граничными условиями, накладываемыми на $F_{m}(\mathbf{x})$ [3].

Очевидно, что для двумерных сверхрешеток, состоящих из периодически расположенных в плоскости $x, y$ квантовых нитей, и одномерных сверхрешеток, образованных из квантовых ям, выращенных в направлении оси $z$, задача определения энергетического спектра сведется к решению соответственно двумерного и одномерного уравнений Шредингера. Эффективные гамильтонианы таких систем будут параметрически зависеть от $\mathbf{K}_{\|}, k_{z}\left(\mathbf{K}_{\|}-\right.$вектор в плоскости $\left.x, y\right)$ и $K_{z}, k_{x}, k_{y}$ соответственно. При переходе к одиночным квантовым нитям и квантовым ямам, когда эффектами туннелирования можно пренебречь, в этих гамильтонианах следует положить соответственно $\mathbf{K}_{\|}=0$ и $K_{z}=0$.

В качестве примера рассмотрим электронные состояния в модельной гетероструктуре с параболической квантовой ямой и гетероструктурах на основе прямозонных полупроводников $\mathrm{A}^{3} \mathrm{~B}^{5}$, содержащих одну квантовую яму, выращенную в направлении [001], образованную в результате замещения атомов в одной подрешетке кристалла. Если период искусственной сверхрешетки равен четному числу монослоев $N$ ( $a_{0} / 2$ - ширина монослоя, $a_{0}$ - период решетки), то задача сводится к решению одномерного уравнения Шредингера [1]. В рамках однозонной $\Gamma_{1}$-модели, не учитывающей поправок от короткодействующей части

Письма в ЖТФ, 2018, том 44, вып. 6 
интерфейсного потенциала, эффективный гамильтониан электрона в $k$-преставлении можно записать в следующем виде [3]:

$$
H\left(k_{i}, k_{j}\right)=\frac{\hbar^{2} k_{i}^{2}}{2 m^{*}} \delta_{i j}+U\left(k_{i}-k_{j}\right) .
$$

Здесь $m^{*}$ - эффективная масса электрона, $k_{i}=(2 \pi / D) i-$ волновой вектор электрона, направленный вдоль оси $z, D=N a_{0} / 2$ - период сверхрешетки, $i=-N / 2 \ldots N / 2-1\left(k_{x}=k_{y}=0\right), U\left(k_{i}-k_{j}\right)-$ Фурье-образ потенциальной энергии $U\left(a_{i}\right)$, заданной в дискретных точках $a_{i}=\left(a_{0} / 2\right) i$ и определяющей пространственное распределение потенциала в гетероструктуре в направлении оси $z$,

$$
U\left(k_{i}-k_{j}\right)=\frac{1}{N} \sum_{i^{\prime}} U\left(a_{i^{\prime}}\right) e^{-i\left(k_{i}-k_{j}\right) a_{i^{\prime}}} .
$$

В качестве модельной тестовой задачи рассмотрим одиночную квантовую яму с параболическим потенциалом $U\left(a_{i}\right)=\left(m^{*} \omega^{2} a_{i}^{2}\right) / 2$, для которой в континуальном приближении известно точное аналитическое решение для собственных волновых функций $F_{n}(z)$ и энергий $E_{n}=\hbar \omega(n+1 / 2)$, где $n=0,1,2, \ldots$ На рисунке (часть $a$ ) представлены результаты численного расчета огибающих функций $F(z)$ для четырех нижних состояний электрона в параболической яме. Для параметров квантовой ямы, приведенных в подписи к рисунку, $a$, относительная погрешность определения энергетического спектра для 50 нижних уровней энергии не превышала $10^{-10 \%}$.

Аналогичные данные для модельных гетероструктур с одиночной прямоугольной и треугольной квантовыми ямами в GaAs приведены соответственно на частях $b$ и $c$ рисунка. Анализ показывает, что учет в эффективном $k p$-гамильтониане гетероструктуры поправок, обусловленных короткодействующей частью интерфейсного потенциала [3], на резком интерфейсе приводит к разрыву огибающей функции $F(z)$ и ее производной, а также к изменению энергетического спектра электрона.

Таким образом, определение энергетического спектра и волновых функций носителей заряда в гетероструктурах в рамках метода эффективной массы сводится к решению системы линейных алгебраических уравнений. Переход же от этой системы к дифференциальному уравнению или к системе дифференциальных уравнений для многозонной $k p$-модели является лишь континуальным приближением этих точных уравнений.

Письма в ЖТФ, 2018, том 44, вып. 6 
Работа выполнена в рамках государственного задания Министерства образования и науки РФ (проектная часть, 16.1750.2017/4.6).

\section{Список литературы}

[1] Ivchenko E.L., Pikus G.E. Superlattices and other heterostructures. Symmetry and optical phenomena. Springer Ser. in Solid-State Sciences. Springer-Verlag, 1997. V. 110.370 p.

[2] Глинский Г.Ф. Полупроводники и полупроводниковые наноструктуры: симметрия и электронные состояния. СПб.: Технолит, 2008. 324 с.; http://www.twirpx.com/file/1014651/

[3] Глинский Г.Ф., Миронова М.С. // ФТП. 2014. Т. 48. В. 10. С. 1359-1369.

[4] Бир Г.Л., Пикус Г.Е. Симметрия и деформационные эффекты в полупроводниках. М.: Наука, 1972. 584 с.

[5] Глинский Г.Ф. Методы теории групп в квантовой механике. СПб.: Изд-во СПбГЭТУ „ЛЭТИ“, 2012. 200 c.; http://www.twirpx.com/file/1014645/ 